\title{
Deducing the Milky Way's Massive Cluster Population
}

\author{
M. M. Hanson ${ }^{1}$, B. Popescu ${ }^{1}$, S. S. Larsen ${ }^{2}$ and V. D. Ivanov ${ }^{3}$ \\ ${ }^{1}$ Department of Physics, University of Cincinnati, Cincinnati, OH 45221, USA \\ ${ }^{2}$ Astronomical Institute, University of Utrecht, Princetonplein 5, 3584 CC Utrecht, NL \\ ${ }^{3}$ European Southern Observatory, Ave. Alonso de Cordova 3107, Santiago 19001, Chile
}

\begin{abstract}
Recent near-infrared surveys of the galactic plane have been used to identify new massive cluster candidates. Follow up study indicates about half are not true, gravitationallybound clusters. These false positives are created by high density fields of unassociated stars, often due to a sight-line of reduced extinction. What is not so easy to estimate is the number of false negatives, clusters which exist but are not currently being detected by our surveys. In order to derive critical characteristics of the Milky Way's massive cluster population, such as cluster mass function and cluster lifetimes, one must be able to estimate the characteristics of these false negatives. Our group has taken on the daunting task of attempting such an estimate by first creating the stellar cluster imaging simulation program, MASSCLEAN. I will present our preliminary models and methods for deriving the biases of current searches.
\end{abstract}

Keywords. Galaxy: stellar content — open clusters and associations: general

What would we see if we could view our whole galaxy? With regards to the stellar clusters, van den Bergh \& Lafontaine (1984) answered this by extrapolating the locally derived luminosity function of star clusters to our whole galaxy. This resulted in an estimate of 100 clusters with $\mathrm{m} \sim 10^{4} \mathrm{M}_{\odot}$ and several clusters of $\mathrm{m} \sim 10^{5} \mathrm{M}_{\odot}$. These early predictions have held up well with time. Using modern, infrared surveys, Ivanov et al. (2009) extended the observed local density of massive clusters to predict $\geqslant 81 \pm 21$ such clusters $\left(>10^{4} \mathrm{M}_{\odot}\right)$ should exist in the Milky Way. Where are they all? Presently one cluster has a mass approaching $10^{5} \mathrm{M}_{\odot}$ (Westerlund 1) and perhaps 10 additional clusters are known with mass $\sim 10^{4} \mathrm{M}_{\odot}$. Is this proof the cluster mass function in the Milky Way steepens or is truncated at the high mass end?

We believe even infrared surveys are plagued with complex biases which limit our ability to estimate what clusters might be missed. Estimating these false negatives is critical to deriving the mass function in the Milky Way. We have created a sophisticated stellar cluster simulation program, MASSCLEAN (Popescu \& Hanson 2009) which when combined with current surveys, will be used to derive the biases of current search methods to differing cluster properties (age, mass, distance, extinction, core density, etc.) and as a function of location in the galaxy. MASSCLEAN will also be used to design more efficient stellar cluster searches.

This material was based upon work supported by the National Science Foundation under Grant No. 0607497 to the University of Cincinnati.

\section{References}

Ivanov, V. D., Messineo, M., Zhu, et al. 2009, A\&SA, submitted

Popescu, B. \& Hanson, M. M. 2009, AJ, submitted

van den Bergh \& Lafontaine 1984, AJ, 89, 1822 